\title{
EL VÍNCULO EXTERNO REMUNERADO DE LA UNIVERSIDAD DE COSTA RICA COMO MEDIO PARA INCREMENTAR EL APORTE INSTITUCIONAL A LA SOCIEDAD
}

\author{
Isabel Cristina Arroyo Venegas \\ Directora de la Escuela de Administración de Negocios \\ de la Universidad de Costa Rica \\ San José, Costa Rica \\ Liana Penabad Camacho \\ Docente de la Universidad Hispanoamericana \\ San José, Costa Rica
}

Recibido 15-IV-2008 • Aceptado 13-V-2008 • Corregido 10-VI-2008

\begin{abstract}
Resumen: Las actividades del Vínculo Externo Remunerado (VER); que ofrece la Universidad de Costa Rica han experimentado un gran impulso y un fuerte crecimiento en la última década y han generado, cada vez más, un mayor impacto en su gestión estratégica y en el aporte que hace la Institución a los distintos sectores de la sociedad.

Si bien existen importantes avances en la gestión del VER-UCR, aún persisten algunas debilidades y limitaciones que llaman a la reflexión y a la decisión de plantear y ejecutar algunos ajustes, cambios e innovaciones que faciliten y promuevan el fortalecimiento en este ámbito de acción del quehacer universitario para finalmente lograr la consecución de la misión institucional.

En congruencia con lo anterior, en este artículo se presenta una propuesta, que busca incrementar el aporte que el VER le genera a la sociedad y al desarrollo de la gestión universitaria; las ideas y acciones para mejorar y fortalecer el VER-UCR se exponen desde cuatro perspectivas o ámbitos: conceptualización, marco estratégico, acciones a nivel operativo y seguimiento y evaluación de resultados.
\end{abstract}

Palabras clave: Vínculo externo, gestión, marco estratégico, indicadores de gestión.

\section{Introducción}

A nivel mundial la educación superior experimenta un periodo de reformas radicales en su estructura de financiamiento y en su gestión para responder adecuadamente a las necesidades que le plantea el entorno. En ese sentido, en Costa Rica la tendencia va en esa dirección, por lo que la actividad de vínculo externo remunerado demanda una mayor atención como un mecanismo estratégico para solventar necesidades del quehacer universitario y de los diferentes sectores que conforman el entorno nacional.

De manera concreta, el impacto que actualmente tiene el Vínculo Externo Remunerado (VER), de la Universidad de Costa Rica (UCR) en su estructura financiera, evidencia las tendencias contemporáneas de esta actividad, pues genera, un aporte relevante en los ingresos totales de la Institución y le permite ofrecer un número significativo de actividades en la 


\begin{abstract}
There is no doubt that External Remunerated Link activities, ERL, offered by the University of Costa Rica have experimented a great promotion as well as a strong growth during the last decade; this has generated a great impact in the institution's management and in the contribution it makes to the different social groups.

Even though important advances in the management of the ERL-UCR exist, it is clear that some weaknesses and limitations still persist and call, both to meditation and decision, to raise and execute some adjustments, changes and innovations that must facilitate and promote the strengthening in this university's range of action, that will, finally, assist the institution in the achievement of its mission.

In line with the stated above, this article presents a proposal, that in general terms, seeks to increase the contribution that ERL generates to the society and the development of the university management. The ideas and actions to improve and strengthen the ERL-UCR are expose from four perspectives or scopes: definition, strategic work frame, actions at the operative level and achievement and evaluation of results.
\end{abstract}

Key words: External link, management, strategic work frame, management indicators. mayoría de las áreas del conocimiento en las que participan diferentes unidades y académicos de la Institución.

En este artículo se rescataron algunas percepciones y criterios, obtenidos mediante un proceso de consulta a diferentes grupos de informantes claves relacionados con el VER, que muestran el rol que tiene esta actividad en el cumplimiento de la misión institucional y, con base en la información que se obtuvo, se expondrá una propuesta que busca fortalecer y redimensionar la gestión en este ámbito, como uno de los ejes prioritarios del quehacer estratégico universitario.

El tema se desarrolla con respaldo en varias secciones que incluyen: el papel del VER-UCR en su gestión estratégica, principales beneficios y limitaciones detectadas y algunas ideas y acciones para mejorar y fortalecer el VER-UCR.

\section{El papel del VER-UCR en el cumplimiento de su misión institucional}

La importancia del VER-UCR se manifiesta en el contexto universitario desde la percepción de distintos grupos involucrados en la formulación y ejecución del marco estratégico institucional sobre el impacto que tienen esas actividades en los resultados globales de la gestión estratégica universitaria.

\subsection{Contexto y percepción}

Por el carácter de instituciones públicas costarricenses, aunque autónomas, propio de las cuatro universidades estatales, las actividades del VER deben sujetarse a un marco jurídico y normativo que puede clasificarse en tres niveles. El primer nivel se refiere a las leyes relacionadas que rigen en el ámbito nacional; el segundo le da sustento a la creación, autonomía y marcos estratégicos de estas Instituciones y un 
tercer nivel que es específico de cada centro de enseñaza superior.

En el entorno costarricense el Plan Nacional para la Educación Superior Universitaria Estatal(PLANES) 2006-2010 (Consejo Nacional de Rectores [CONARE], Oficina de Planificación de la Educación Superior [OPES], 2005), se fundamenta en cinco ejes: pertinencia e impacto; calidad; cobertura y equidad; ciencia, tecnología e innovación; y gestión. El eje Pertinencia e Impacto, destaca en su definición y lineamientos estratégicos la importancia de la vinculación y nuevas formas de relacionarse con el sector nacional e internacional, con el fin de responder a las demandas del país y a las del nuevo orden mundial.

En la Universidad de Costa Rica, el carácter público y el enfoque humanista, están definidos por los principios que le ordena su Estatuto Orgánico. Especialmente el Título I, Artículo 1, define a la Universidad como:

...una institución autónoma de cultura superior, constituida por profesores, estudiantes y funcionarios administrativos dedicada a la enseñanza, la investigación, la acción social, el estudio, la meditación, la creación artística y la difusión del conocimiento (Universidad de Costa Rica, Oficina Jurídica, 2000, p. 22).

En su Artículo 3, con el propósito de:

...obtener las transformaciones que la sociedad necesita para el logro del bien común, mediante una política dirigida a la consecución de una verdadera justicia social, del desarrollo integral, de la libertad plena y de la total independencia de nuestro pueblo (Universidad de Costa Rica, Oficina Jurídica, 2000, p. 22).

La Universidad desde sus inicios, hace más de seis décadas, despliega una serie de esfuerzos de vinculación externa con diferentes grupos y sectores de la sociedad costarricense, bajo dos modalidades de financiamiento; una de ellas es la Vinculación Externa No Remunerada y la otra es la Vinculación Externa Remunerada. La primera se financia a través de un presupuesto propio y la segunda con la contribución de terceros; desde esta perspectiva, en la Vinculación Externa No Remunerada la contraprestación entre las partes se rige por el interés que persigue la obtención de un fin específico, mientras que en la Vinculación Externa Remunerada la contraprestación se rige por el beneficio de ambas partes.

En la Universidad de Costa Rica el VER, se ofrece desde las distintas vicerrectorías y unidades académicas en la mayoría de las áreas y su gestión financiera se canaliza por medio de la Oficina de Administración Financiera de la Universidad (OAF) y de la Fundación de la Universidad de Costa Rica para la investigación (FUNDEVI). Con base en las cifras financieras obtenidas en el año 2006, los ingresos generados por el VER-UCR representaron un $72 \%$ del total de ingresos reportados por el VER de las cuatro universidades públicas.

Con el fin de obtener una percepción general del Vínculo Externo Remunerado -a lo interno de la Universidad-se realizó un proceso de consulta por medio de entrevistas a 14 autoridades (de nivel superior e intermedio) de dependencias o unidades académicas con un rol permanentemente activo en este ámbito del quehacer universitario.

A partir de dichas entrevistas, el concepto de Vínculo Externo es definido como la prestación de servicios a cambio de una remuneración o pago y en parte como una relación contractual entre la Universidad y terceras personas. Algunos lo conciben, también, como una opción para transferir conocimiento desde la Universidad a los distintos sectores de la comunidad, que genera un beneficio social y de cooperación solidaria; otros lo consideran una actividad de servicio extraordinario cuyos excedentes deben financiar el quehacer académico institucional pero en definitiva sin fines de lucro.

En relación con las políticas existentes para la gestión del VER, en general hay un claro conocimiento del marco normativo que regula estas actividades, algunos 
entrevistados coinciden en que todavía, esta no es suficiente y presenta cierta ambigüedad. También, se evidenció que, solo en unos pocos casos, existen políticas y directrices propias, algunas de ellas relacionadas con la distribución de los recursos que se generan.

Es importante aclarar, que la mayoría de las autoridades consultadas han jugado un doble rol en las actividades de VER, tanto en su dirección y coordinación, como participando o ejecutando proyectos o actividades dentro de esta modalidad.

Sobre el enfoque humanista y la responsabilidad social del VER, se comparte el criterio de que no todas las actividades que actualmente se desarrollan por medio de VER, responden en la forma debida a este enfoque, que cimenta los ejes fundamentales de la gestión universitaria, aunque sí se reconoce que en algunas unidades académicas se concibe y se ejecuta de acuerdo con estos principios. Por lo tanto, puede afirmarse que responde parcialmente a una visión solidaria demostrada, por ejemplo, con el aporte al Fondo de Desarrollo Institucional.

Con respecto a la participación del VER dentro de la estructura de financiamiento de la UCR, la mayoría de las autoridades consultadas señalan que el VER debe concebirse como una fuente de ingresos complementaria al presupuesto que debe otorgar el Estado, y como una vía necesaria para financiar una oferta de productos y servicios de calidad, que no sería posible solo con el aporte estatal.

También se realizó una consulta, por medio de entrevistas, a 18 usuarios y beneficiarios que contrataron, durante el año 2006, diversos servicios y productos por medio del VER; de estas personas consultadas, siete, con una posición solo de usuarios que contrataron para terceras personas y 11 en la doble posición de usuarios-beneficiarios, ya que contrataron para recibir directamente el beneficio. La selección de este grupo responde al criterio de la importancia relativa que representan algunas áreas en la generación de los ingresos totales del VER.

Catorce de los 18 entrevistados manifestaron conocer algunas de las actividades que desarrolla la UCR a través de la Vinculación Remunerada, aunque quedó claro que ninguno evidenció un conocimiento integral de la amplitud de la oferta del VER, así como que no existe una clara diferenciación entre lo que es una actividad de vinculación remunerada o una actividad de vinculación no remunerada. Los usuarios beneficiarios de los posgrados con financiamiento complementario no los consideran una actividad de Vinculación Remunerada, pues los ven como parte de la oferta académica de la Universidad.

A pesar de que el 59\% de los servicios contratados fueron calificados como buenos, otros seis servicios fueron calificados como regulares y tres como deficientes; estos últimos en Posgrados, programas de capacitación en el área de educación y venta de materiales en la Escuela de Artes Plásticas. En relación con los costos de los servicios o productos contratados, un $30 \%$ los consideran altos y un $70 \%$ los cree razonables.

\subsubsection{Visualización del VER en el Marco Estratégico-UCR}

Si bien es cierto, que desde el Estatuto Orgánico se plantea una necesidad de que la Institución desarrolle actividades de vinculación con los diferentes sectores de la sociedad, y a pesar de que existen dentro de las políticas un eje estratégico que se puede relacionar con el VER, así como un eje crítico y cuatro objetivos estratégicos en el Plan de Gestión y Proyectos 2004-2008; es cierto también, que todavía dentro de este marco estratégico no se plantea o se define claramente una visión que plasme el concepto, los alcances y la trascendencia que representan todas las actividades del VER dentro y fuera de la Universidad. 
Se expone en este marco de manera indirecta, la prioridad en torno a la vinculación universitaria en general con distintos actores de la sociedad, sin que quede clara la necesidad de una coexistencia entre los dos tipos de vinculación que puede generar la institución: remunerada y no remunerada; que juntas constituyen la mejor opción para lograr un desarrollo humano y una mejora en la calidad de vida de los costarricenses, pese a las limitaciones presupuestarias a las que históricamente se ha enfrentado la Universidad.

Por lo tanto, puede considerarse que existen vacíos en la visualización del VER dentro de la dinámica universitaria y que se requiere un replanteamiento a nivel de políticas y objetivos estratégicos, ya que en definitiva, no hay un plan estratégico del VER-UCR que integre los planes de las distintas instancias y los alinee con el Plan Institucional.

\subsubsection{Impacto del VER-UCR en los resultados de su gestión estratégica}

En este aparte se presenta un análisis principalmente de naturaleza cuantitativa, que pretende resaltar el impacto del VER en la gestión de la UCR. Se entenderá el término impacto como la repercusión o influencia importante que generan todas las actividades de la vinculación remunerada, sobre los resultados financieros de la Institución y en general sobre la gestión de las distintas Unidades Académicas que la conforman, así como sobre los distintos grupos de la sociedad que reciben los beneficios producto de dichas actividades. El impacto del VER-UCR en los resultados de su gestión se puede visualizar desde dos perspectivas: la financiera y la social.

La información expuesta en este punto se circunscribe a la actividad del VER durante el periodo 2006 y proviene de reportes, informes y estados financieros oficiales de la Universidad de Costa Rica y de FUNDEVI.

\subsubsection{Impacto Financiero}

En el ámbito financiero, el impacto del VER se puede cuantificar a partir de tres parámetros: ingresos, egresos -que incluye algunos aportes a la gestión de proyectos de vinculación no remunerada- e inversiones, que constituyen egresos capitalizables; es decir, aportes al quehacer universitario en todos sus ámbitos de acción.

Los ingresos totales del VER desarrollado por la Universidad de Costa Rica -independientemente de la instancia que administró los recursos- generó ingresos totales por $\$ 15.551 .905 .681$, de los cuales un $74 \%$ se manejó por medio de OAF y un $26 \%$ por medio de FUNDEVI. La Vicerrectoría de Investigación aportó el $42 \%$, Acción Social un 33\%, Docencia un $15 \%$ y el restante $10 \%$ lo aportaron las Sedes Regionales, Dirección Superior, Vida Estudiantil y Administración.

Los egresos totales del VER, en ese mismo periodo, sumaron $\phi 11.487 .779 .612$, de éstos $\phi 10.157 .039 .322$ correspondieron a egresos operativos y $\phi 1.330 .740 .290$ a egresos capitalizables o inversiones; la mayor participación en ellos la tuvo Acción Social con un $41 \%$, seguida de Investigación con un $38 \%$ y Docencia con un $14 \%$.

Al analizar la composición de esos egresos es posible determinar; que un $44 \%$ del total de los egresos operativos fue generado por Acción Social, un 36\% por Investigación y un 13\% por Docencia; mientras que, la participación en el total de las inversiones o egresos capitalizables fue de un 57\% para Investigación, un $23 \%$ para Docencia y un $12 \%$ para Acción Social. En definitiva, Investigación fue la Vicerrectoría que más invirtió y Acción Social la que produjo el mayor monto de gastos operativos.

Con respecto a los resultados netos y según lo muestra el siguiente cuadro, el VER consolidado aportó en total excedentes por \$4.064.126.069, manteniendo siempre la Vicerrectoría de Investigación el mayor aporte en este total. 
Cuadro 1

Vínculo Externo Remunerado, Universidad de Costa Rica

Resultados netos

Consolidado OAF-FUNDEVI

Periodo 2006

(en colones)

\begin{tabular}{|c|c|c|c|c|}
\hline Área & $\begin{array}{l}\text { Ingresos } \\
\text { totales }\end{array}$ & $\begin{array}{l}\text { Egresos } \\
\text { totales }\end{array}$ & $\begin{array}{l}\text { Excedentes } \\
\text { netos }(* *)\end{array}$ & $\begin{array}{c}\text { Margen neto } \\
\%\end{array}$ \\
\hline Investigación & 6.551 .561 .147 & 4.382 .097 .524 & 2.169 .463 .623 & 33 \\
\hline Acción Social & 5.095 .791 .134 & 4.672 .967 .864 & 422.823 .270 & 8 \\
\hline Dirección Superior & 420.344 .864 & 136.102 .617 & 284.242 .247 & 68 \\
\hline Docencia & 2.299 .283 .445 & 1.630 .500 .245 & 668.783 .200 & 29 \\
\hline Vida Estudiantil & 183.177 .261 & 47.393.178 & 135.784 .083 & 74 \\
\hline Administración & 1.361 .354 & 1.009 .932 & 351.422 & 26 \\
\hline Desarrollo Regional (*) & 999.842 .726 & 617.441 .685 & 382.401 .041 & 38 \\
\hline Otros & $543.750,00$ & 266.567 & 277.183 & 51 \\
\hline TOTALES & 15.551 .905 .681 & 11.487 .779 .612 & 4.064.126.069 & 26 \\
\hline
\end{tabular}

$\left(^{*} \quad\right.$ En este renglón se incluyen solo los ingresos y egresos de las Sedes Regionales que son administrados por OAF, pues los que administra FUNDEVI están incluidos en las distintas áreas de acuerdo con su naturaleza.

$(* *) \quad$ Estos excedentes deberán interpretarse como un DISPONIBLE, pues la conclusión o cierre de cada actividad no coincide necesariamente con el cierre del periodo.

Fuente: Universidad de Costa Rica. Informes financieros de OAF y FUNDEVI.

Por último, si se incorporan los resultados del Vínculo Externo Remunerado administrado por FUNDEVI al Estado de Ingresos y Egresos de la UCR (Universidad de Costa Rica, 2006d), para el periodo 2006, se desprende que la participación de estas actividades en los ingresos totales pasa de un $13 \%$ a un $17 \%$. Los egresos asociados incrementan su peso en los ingresos totales, al cambiar de un $10 \%$ a un 14\%. En consecuencia, el neto disponible se incrementa en un $5,3 \%$ que equivale a ф590.915.574; en los gráficos 1, 2, 3 y 4 se reflejan estas relaciones.

\subsubsection{Impacto social}

Dadas algunas limitaciones y debilidades que se detectaron en la revisión documental, en lo que corresponde a sistemas de información, con los reportes que se emiten en los distintos niveles y dependencias solo es posible realizar una evaluación en términos globales, aunque con indicadores claros de los alcances en este ámbito.

Si se analizan los datos relacionados con el número de proyectos de vinculación remunerada vigentes al 31 de diciembre de 2006 y que se presentan en Cuadro 2, sin duda alguna se evidencia la cantidad de iniciativas que surgen desde las distintas áreas de gestión y en las que participan muchas unidades académicas de todas las áreas del conocimiento. Concretamente, se desarrollaron 957 -un 52\% administrados por OAF y un $48 \%$ por FUNDEVI- siendo la Vicerrectoría de Investigación la que mayor participación tuvo con un 43,7\%, seguida de Acción Social con un 31,1\% y 
Gráfico 1

Participación del Vínculo Externo Remunerado en los ingresos UCR

Total de ingresos sin FUNDEVI

Periodo 2006

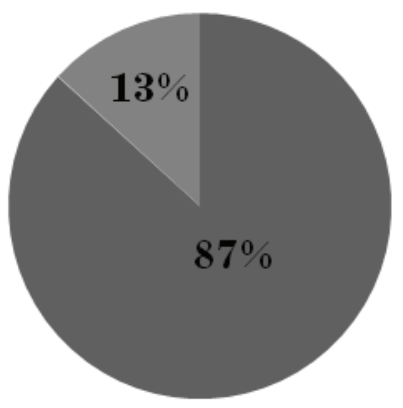

- Fondos corrientes

- Vínculo remunerado

Gráfico 3

Participación del Vínculo Externo Remunerado en los egresos UCR

Total de egresos sin FUNDEVI

Periodo 2006

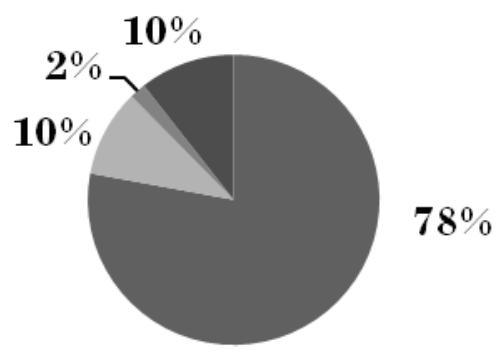

\begin{tabular}{|ll|}
\hline - Fondos corrientes & -Fondos préstamos \\
- Planta física & - Vínculo remunerado
\end{tabular}

\section{Gráfico 2}

Participación del Vínculo Externo Remunerado en los ingresos UCR Total de ingresos con FUNDEVI Periodo 2006

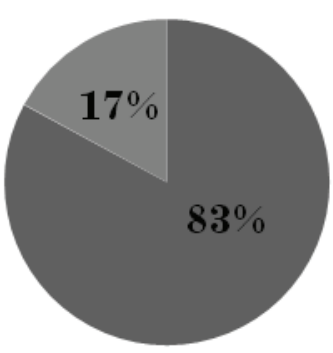

- Fondos corrientes

- Vínculo remunerado

Gráfico 4

Participación del Vínculo Externo Remunerado en los egresos UCR

Total de egresos con FUNDEVI

Periodo 2006

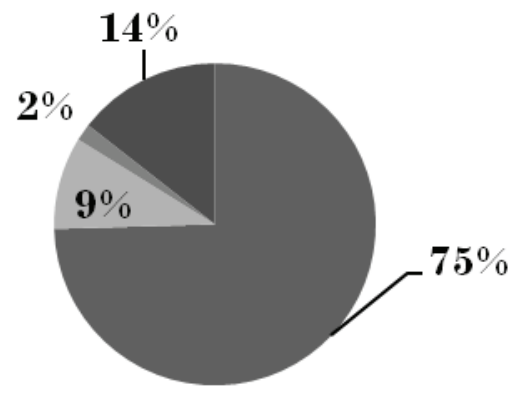

- Fondos corrientes

Fondos préstamos

Planta física

- Vínculo remunerado 
Cuadro 2

Número de proyectos de Vinculación Externa Remunerada por programa y por administrador financiero vigentes al 31/12/2006

\begin{tabular}{|c|c|c|c|c|c|c|c|c|c|c|}
\hline 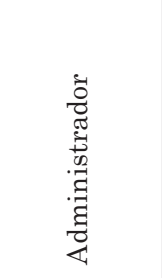 & 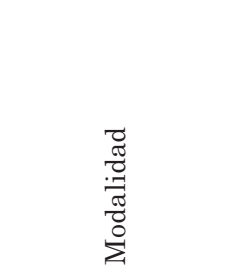 & 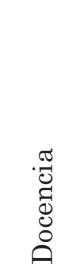 & 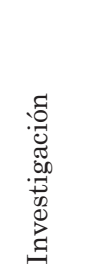 & 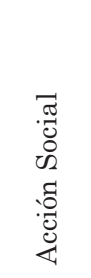 & 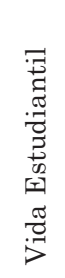 & 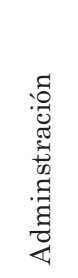 & 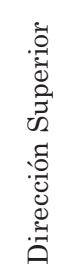 & 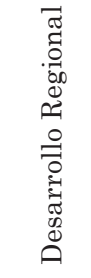 & 总 & 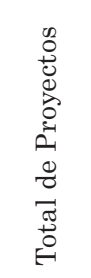 \\
\hline \multirow{2}{*}{$\mathrm{OAF}$} & Total OAF & 144 & 185 & 93 & 16 & 4 & 16 & 44 & 0 & 502 \\
\hline & $\begin{array}{l}\text { Total relativo } \\
\text { OAF }\end{array}$ & $29 \%$ & $37 \%$ & $19 \%$ & $3 \%$ & $1 \%$ & $3 \%$ & $9 \%$ & $0 \%$ & $100 \%$ \\
\hline \multirow{2}{*}{ FUNDEVI } & Total FUNDEVI & 4 & 233 & 204 & 4 & 1 & 4 & 0 & 5 & 455 \\
\hline & Total relativo & $1 \%$ & $51 \%$ & $45 \%$ & $1 \%$ & $0 \%$ & $1 \%$ & $0 \%$ & $1 \%$ & $100 \%$ \\
\hline \multirow{4}{*}{ TOTALES } & $\begin{array}{l}\text { Total Proyecto } \\
\text { VER }\end{array}$ & 148 & 418 & 297 & 20 & 5 & 20 & 44 & 5 & 957 \\
\hline & Total relativo & $15 \%$ & $44 \%$ & $31 \%$ & $2 \%$ & $1 \%$ & $2 \%$ & $5 \%$ & $1 \%$ & $100 \%$ \\
\hline & $\begin{array}{l}\% \text { Administrado } \\
\mathrm{OAF}\end{array}$ & $97 \%$ & $44 \%$ & $31 \%$ & $80 \%$ & $80 \%$ & $80 \%$ & $100 \%$ & $0 \%$ & $52 \%$ \\
\hline & $\begin{array}{l}\% \text { Administrado } \\
\text { FUNDEVI }\end{array}$ & $3 \%$ & $56 \%$ & $69 \%$ & $20 \%$ & $20 \%$ & $20 \%$ & $0 \%$ & $100 \%$ & $48 \%$ \\
\hline
\end{tabular}

Fuente: Universidad de Costa Rica. Informes de OAF y FUNDEVI.

Docencia con un $15,4 \%$. Con esas cifras y porcentajes globales se visualiza, en gran medida, la magnitud de un impacto social desde la perspectiva de la generación y transmisión de conocimiento, producto de la vinculación remunerada y que se convirtió en beneficio directo para diversos grupos de la comunidad nacional.

En forma concreta, en el Cuadro 3, se reflejan algunas cifras que corresponden a la participación de las diferentes áreas -de gestión y académicas- en las actividades de vinculación remunerada, de lo cual se puede deducir que las personas o grupos beneficiados con el VER-UCR, pertenecen a todos los sectores de la sociedad.
En síntesis, el impacto social del VER-UCR es evidente, no solo por el número de proyectos y por la participación de las distintas áreas de gestión y del conocimiento, sino también por el fuerte apoyo que representa en la gestión estratégica institucional que procura el desarrollo de los distintos grupos y sectores, con prioridad en los más vulnerables o necesitados.

\section{Principales beneficios y limitaciones del VER-UCR}

Con base en la información que se obtuvo en el proceso de consulta a los grupos 
Cuadro 3

Vínculo Externo Remunerado-OAF

Ingresos por área

Período 2006

(en colones)

\begin{tabular}{|c|c|c|c|c|}
\hline Área & Ingresos & $\begin{array}{c}\% \\
\text { Participación }\end{array}$ & $\begin{array}{c}\# \\
\text { Proyectos }\end{array}$ & $\begin{array}{c}\% \\
\text { Participación }\end{array}$ \\
\hline Acción social & $3.727 .745 .142,78$ & 32,4 & 78 & 15,5 \\
\hline Investigación & 1.968.679.708,53 & 17,1 & 89 & 17,7 \\
\hline Centros e institutos de investigación & $1.458 .519 .724,56$ & 12,7 & 99 & 19,7 \\
\hline Desarrollo regional & $999.842 .726,65$ & 8,7 & 43 & 8,6 \\
\hline Sistema de Estudios de Posgrado & $642.783 .956,88$ & 5,6 & 8 & 1,6 \\
\hline Docencia & $488.366 .905,77$ & 4,2 & 41 & 8,2 \\
\hline Dirección Superior & $290.509 .457,74$ & 2,5 & 15 & 3,0 \\
\hline Vida estudiantil & $175.407 .469,95$ & 1,5 & 17 & 3,4 \\
\hline Administración & $1.316 .444,69$ & 0,0 & 2 & 0,4 \\
\hline Salud & 1.045.566.009,37 & 9,1 & 30 & 6,0 \\
\hline Ciencias Sociales & $335.444 .125,83$ & 2,9 & 38 & 7,6 \\
\hline Ingenierías y Arquitectura & $272.064 .572,75$ & 2,4 & 25 & 5,0 \\
\hline Ciencias Básicas & $54.908 .620,90$ & 0,5 & 7 & 1,4 \\
\hline Artes y Letras & $45.380 .329,11$ & 0,4 & 6 & 1,2 \\
\hline Estudios Generales & $1.710 .603,49$ & 0,01 & 4 & 0,8 \\
\hline TOTAL & $11.508 .245 .799,00$ & 100,0 & 502 & 100,0 \\
\hline
\end{tabular}

Fuente: Universidad de Costa Rica. Informes Financieros de OAF.

de informantes clave, involucrados con el VER-UCR, se rescatan a continuación, los principales beneficios, limitaciones $\mathrm{y}$ principales metas que debe plantearse el VER-UCR.

\subsection{Sobre los beneficios y limitaciones del VER}

De acuerdo con la percepción de las autoridades y usuarios-beneficiarios consultados, el VER genera importantes beneficios para la Institución y sus dependencias, así como para las personas o grupos que reciben el beneficio de esta actividad, tanto en el ámbito financiero como social. Entre ellos se destacan:

- Permite la obtención de recursos financieros complementarios.

- Fortalece la relación UniversidadSociedad.

- Fortalece la imagen de la Universidad.

- $\quad$ Ayuda a mejorar la gestión universitaria, así como su infraestructura.

- Constituye una vía para que académicos y estudiantes conozcan la realidad nacional y lleven a la práctica sus conocimientos.

- Desarrollo personal y familiar. 
- Realización profesional.

- Oportunidad de acceso al conocimiento para el sector laboral.

- Generación desolucioneseimportantes aportes al sector laboral.

- Promueve la educación continua.

Asimismo, se destacan algunas limitaciones o debilidades del VER, entre las que se encuentran:

- La ausencia de una cultura de relación estrecha Universidad-Sociedad, reflejada en algunos grupos universitarios.

- No hay una exigencia de que todas las actividades del VER generen en primer término una rentabilidad académica o de investigación y aun existiendo ésta se dificulta su medición.

- Aún no existe un planteamiento claro sobre el VER y sus mecanismos de regulación.

- Algunos horarios y sistemas de pago.

- $\quad$ Estado de la infraestructura en algunas áreas.

- Escasa información sobre la oferta de servicios.

- $\quad$ Altos costos para algunos de los grupos que acceden a ciertos servicios o productos.

\subsection{Sobre las metas que debe fijarse el VER-UCR}

De acuerdo con el criterio de los grupos consultados, las metas que debe fijarse el VER son:

- La construcción de una normativa clara e integral.

- Conocimiento de las necesidades prioritarias y expectativas de los usuarios.

- Elaboración de un plan estratégico institucional para el VER.

- Introducir innovación en sus mecanismos de administración.
- Plantear una oferta de bienes y servicios coherente e integral, dirigidos hacia la autogestión y el aprovechamiento de la interdisciplinaridad y a la promoción de actividades innovadoras en el área tecnológica y en la concepción de oportunidades de negocios.

- $\quad$ Promover un mejor ambiente intrauniversitario para la gestión de VER.

- Aumentar el aporte del VER en el presupuesto universitario.

- Generar mecanismos de evaluación y seguimiento para todas las actividades.

- Crear cursos de preparación para el aprendizaje virtual.

- Oferta permanente de cursos orientados al segmento de los jóvenes.

\section{Algunas ideas y acciones para mejorar y fortalecer el VER-UCR}

Con fundamento en toda la información recopilada y el respectivo análisis, se presenta de seguido una propuesta que incluye algunos cambios, estrategias y acciones, que buscan solventar las limitaciones y debilidades encontradas, con el propósito de fortalecer la gestión del VER-UCR. Este planteamiento se abordará desde cuatro perspectivas: la conceptual, la estratégica, la operativa y la de seguimiento y control. Por medio de ellas, se pretende ofrecer opciones con un enfoque integral, que permitan la articulación y el mejor aprovechamiento de las oportunidades y recursos disponibles en este ámbito del quehacer universitario.

\subsection{Conceptualización del VER-UCR}

Se debe interpretar el concepto de Vinculación Externa como todas aquellas actividades desarrolladas por la Universidad 
para responder a las necesidades planteadas por diversos grupos sociales, distintos a los que conforman la comunidad universitaria; es decir, académicos, estudiantes y funcionarios administrativos.

Entonces, al retomar las diferentes definiciones o percepciones sobre el VER que se manejan en los distintos niveles o ámbitos de acción universitarios y al contrastarlos con la realidad que le corresponde atender a la Institución, desde sus actividades sustantivas y desde la actividad de vinculación remunerada, quedan claros los siguientes componentes que deben delimitar este concepto:

- Proceso de intercambio entre la Universidad y distintos actores de la sociedad.

- Conjunto de relaciones entre la Universidad y distintos sectores de la sociedad que contribuyen al desarrollo de todas las partes involucradas.

- Vía de cooperación solidaria con la sociedad.

- $\quad$ Actividad derivada del quehacer académico sustantivo universitario.

- Actividades que no forman parte de las ofertas académicas para la preparación de profesionales.

- Venta de bienes y prestación remunerada de servicios con el fin primordial de contribuir al logro del equilibrio social.

Por lo tanto se puede definir Vínculo Externo Remunerado como: Un proceso solidario de intercambio entre la Universidad y distintos actores de la sociedad, que permite generar un conjunto de actividades -derivadas del quehacer académico sustantivo universitario- que contribuyen con el desarrollo y el equilibrio social, por medio de la venta de bienes y prestación remunerada de servicios diferentes de la oferta académica institucional para formar profesionales.

Bajo esta conceptualización, se deben incluir dentro de Vínculo Externo
Remunerado las siguientes actividades: Cursos, seminarios o talleres de actualización y capacitación contratados; investigación contratada o con financiamiento complementario; servicios contratados: de análisis, laboratorio, alquiler de bienes, técnicos e informáticos; consultorías y asesorías; venta de productos y subproductos; procesos de certificación y normalización; transferencia de conocimientos por licenciamientos; actividades artísticas y deportivas remuneradas; intercambio académico remunerado o cualquier otro servicio o actividad remunerados derivados del quehacer universitario y no contemplados en las categorías anteriores.

Por consiguiente, las actividades que deberán excluirse de esta categoría son: posgrados con financiamiento complementario; actividades universitarias regulares de docencia financiadas con fondos asignados por medio de Leyes de la República; venta de materiales y productos que demandan los estudiantes regulares de la Universidad; prestación de servicios a estudiantes regulares de la Universidad donde media algún tipo de ingresos para la Institución; convenios y relaciones contractuales que proporcionen fondos para financiar necesidades de estudiantes y profesores regulares de la Institución; cualquier otra actividad o relación que no implique una contraprestación que genere beneficios económicos recíprocos para la Universidad $\mathrm{y}$ terceras personas.

\subsection{Un Marco Estratégico para el VER-UCR}

Para fortalecer la gestión del VER que desarrolla la UCR, debe asegurarse -en primer término- que a nivel de Marco Estratégico y en lo que corresponde a ejes, políticas, objetivos y estrategias queden claramente evidenciados; la importancia y los alcances de este ámbito del quehacer universitario, en el cumplimiento de la Misión Institucional. Por lo tanto y a partir 
de las políticas y directrices emitidas por el Consejo Universitario para el 2008 y al plan de gestión de la Rectoría, se sugiere incorporar los siguientes cambios:

A nivel de políticas y objetivos:

En el Eje 4 Fortalecimiento de la Vinculación con la comunidad nacional e internacional, de las Políticas y Directrices para el 2008, se estima necesario el replanteamiento de la política general, con el fin de alinear toda la gestión de vinculación externa con el Plan Nacional de desarrollo (PND) y a la vez destacar la importancia de los tipos de vinculación que le corresponde atender a la Institución, para que quede planteada de la siguiente manera:

La Universidad de Costa Rica, por medio de sus labores sustantivas y actividades de vinculación externa -remunerada y no remunerada- y en línea con el Plan Nacional de Desarrollo fortalecerá las condiciones para la interacción con la comunidad nacional e internacional, con el fin de ser una institución que contribuya a la construcción de una sociedad inclusiva, próspera, justa y humanista.

En ese mismo Eje y como consecuencia de su política general, se recomienda adicionar la siguiente política específica:

Promoverá el desarrollo de actividades de Vinculación Externa Remunerada con todos los sectores de la sociedad como mecanismo para potenciar los alcances y beneficios del Vínculo Externo No Remunerado a favor de los grupos más vulnerables del país.
- En el Eje Crítico Proyección Social del Plan de Gestión de la Rectoría, es necesario introducir objetivos estratégicos que dejen claro el papel fundamental que tienen las actividades de vinculación externa (remunerada y no remunerada) en el cumplimiento de la misión social que se le ha encomendado a la Institución. Se proponen los siguientes:

a. Crear una oferta de actividades de vinculación externa, remunerada y no remunerada, alineada con el Plan Nacional de Desarrollo que responda eficazmente a las necesidades y solución de problemas planteados por los diferentes sectores de la comunidad nacional.

b. Potenciar el desarrollo de las actividades de Vinculación Externa Remunerada con el propósito de generar un mejor soporte financiero que permita fortalecer las actividades de Vinculación Externa No Remunerada para los grupos más vulnerables del país.

A nivel de estrategias

En congruencia con las políticas y objetivos que se han propuesto para fortalecer el Vínculo Externo Remunerado de la UCR, se han identificado algunos ámbitos o ejes de acción sobre los que se proponen las siguientes estrategias generales:

Gestión conjunta desde CONARE: Para la introducción, en el marco Estratégico de PLANES 2006-2010, de los cambios que se requieren para destacar la importancia y alcances del VER, así como el concepto de lo que significa y representa este ámbito en el fortalecimiento de la relación Universidad-Sociedad. Esta gestión conjunta podría, también, promover 
una reforma a la Ley de Fundaciones que permita la clara diferenciación de las fundaciones que pertenecen a las universidades públicas y una propuesta de ajustes a las distintas normativas que rigen el VER de las cuatro universidades públicas orientada a equiparar los marcos estratégicos, principalmente en lo referido al principio de solidaridad que debe prevalecer en el manejo financiero de los recursos que se generan en este ámbito.

Diseño de un Plan Estratégico para el VER-UCR: Se estima conveniente que los ejes que sustenten el Plan Estratégico del VER-UCR, estén alineados con los ejes del PND que esté vigente y con los que soporta el Plan Estratégico Institucional.

Por lo tanto, se sugiere elaborar un primer Plan para el periodo 2008. 2010 que se sustente en los siguientes ejes estratégicos:

- Desarrollo de las capacidades humanas.

- Distribución equitativa de oportunidades.

- Estímulo y crecimiento económico.

- Armonía con el ambiente.

- Uso racional y solidario de los recursos.

- Desarrollo social y cultural.

- Tecnología e Innovación.

- Pertinencia.

- Alianzas y cooperación internacionales.

Este Plan podría considerar dentro de su Marco Estratégico los siguientes componentes:

- Misión: Contribuir con las transformaciones, el desarrollo y el equilibrio que requiere la sociedad mediante una oferta pertinente de bienes y servicios -derivados del quehacer universitario- que permita, de forma equitativa y solidaria, la generación de oportunidades y actividades de vinculación externa no remunerada para los diferentes sectores y grupos de la comunidad nacional e internacional.

- Visión: Alcanzar el liderazgo en la innovación y generación de aportes al desarrollo nacional mediante una oferta solidaria y sostenible de bienes y servicios derivados del quehacer universitario.

- Valores: Solidaridad, equidad, innovación, pertinencia, transparencia

- Objetivos estratégicos:

- Promover el desarrollo de las personas mediante procesos de capacitación y actualización para el mejoramiento de su calidad de vida.

- Promover el desarrollo y crecimiento de los diferentes sectores sociales en armonía con el medio ambiente.

- Fomentar el trabajo interdisciplinario a partir de la articulación de las distintas áreas del quehacer universitario para lograr respuestas integrales y pertinentes a las necesidades sociales.

- Fomentar la generación del conocimiento mediante la investigación para el fortalecimiento de la academia y la solución de 
los problemas planteados por los distintos grupos de la comunidad.

- $\quad$ Promover la articulación con distintos sectores de la comunidad nacional e internacional, por medio de alianzas estratégicas que permitan una mayor accesibilidad a los beneficios derivados del quehacer académico.

- Promover la innovación como respuesta a las necesidades sociales mediante el fortalecimiento de las tecnologías.

- Estimular el uso racional de los recursos por medio de una gestión clara y transparente para la generación de una actividad de vinculación eficiente y solidaria.

Generar fuentes de financiamiento por medio de relaciones e intercambios justos que potencien actividades de Vínculo Externo No Remunerado para grupos vulnerables de la sociedad.

Ajustes a normativa-UCR: con esta estrategia se pretende que -a partir de una propuesta de la Rectoríael Consejo Universitario incorpore las modificaciones necesarias a la normativa relacionada con el VER, de tal forma que se fortalezcan los lineamientos para la vinculación con el sector externo y se emita un reglamento que regule integralmente la administración de los recursos generados mediante el VER.

Estructura de apoyo al VER: esta estrategia contempla la creación de una estructura concebida como un dependencia que integre tanto la vinculación remunerada como la no remunerada en un esfuerzo para asegurar el balance, mutuo apoyo y complementariedad que debe existir entre ambas, para brindarle a la sociedad una oferta pertinente y de amplio acceso a los distintos grupos que la conforman, independientemente de su nivel, localización o capacidad financiera. Los ámbitos de gestión de esta estructura se concretarían a: Planificación y Fomento del Vínculo Externo, apoyo y asesoría en la formulación y promoción de las actividades, apoyo y seguimiento a la ejecución de dichas actividades y la medición de resultados a partir de la integración que incorpore todas las actividades y análisis de la información y la emisión de informes de gestión parciales o institucionales.

Fortalecimiento de FUNDEVI: se estima que una buena estrategia para fortalecer el Vínculo RemuneradoUCR es el fortalecimiento de FUNDEVI. Esto se podría lograr dotándola, por un lado, de todos los recursos necesarios para ampliar su capacidad de gestión y mejorar los servicios que presta y, por otro, de las políticas y resoluciones que se requieran para dejar clara la relevancia del papel que juega dentro de la gestión universitaria.

Plan de divulgación, comunicación y promoción del VER: con el propósito de potenciar la gestión del VER y fomentar una cultura que promueva y valore las fortalezas y los alcances de estas actividades y así erradicar los paradigmas y prejuicios que han limitado este importante ámbito de la gestión universitaria; se considera necesaria la elaboración y puesta en marcha de un Plan de 
divulgación y comunicación a nivel institucional, dirigido a dos segmentos: la comunidad universitaria y la comunidad nacional e internacional.

\subsection{Acciones de mejora a nivel operativo}

Como complemento a las acciones que deberán desarrollarse para ejecutar las distintas estrategias que se han propuesto en el Marco Estratégico del VER, se considera necesario darle prioridad a algunas acciones de mejora, que se derivan de las principales limitaciones encontradas al analizar el Marco Operativo, en que actualmente se lleva a cabo la gestión del VER. De manera específica, deberán hacerse ciertos ajustes y cambios en la captura e integración de la información relacionada con todas las actividades de vinculación externa, introducir algunas modificaciones en los procesos de registro contable financiero y ajustar las guías para informes de las actividades de vinculación externa con el propósito de generar una mayor y adecuada retroalimentación y rendición de cuentas.

\subsection{Seguimiento y evaluación de resultados}

Como etapa final de la propuesta desarrollada en este capítulo, se estima necesaria y de gran importancia la implementación de un proceso de seguimiento y evaluación de resultados para la gestión del VER, con el propósito de generar la retroalimentación requerida, para la toma de decisiones eficientes y la mejora continua, así como para respaldar la rendición de cuentas ante los distintos grupos internos y externos de la Universidad, en cumplimiento del principio de transparencia y como estrategia para evidenciar el rol y el impacto del VER-UCR en el desarrollo socioeconómico del país.
Particularmente, para el seguimiento, se sugieren entrevistas, grupos focales e informes de análisis en los que participen los distintos grupos involucrados con el VER.

Para la evaluación de resultados y rendición de cuentas, se sugieren dos tipos de acciones:

encuestas, con los distintos grupos involucrados.

indicadores de gestión, para favorecer una evaluación sistematizada apoyada especialmente en el estudio de datos cuantitativos. Estos indicadores se pueden plantear desde cuatro perspectivas de resultados: generales, académicos, sociales y financieros. A manera de ejemplo se aportan dos indicadores para cada uno de los cuatro ámbitos de gestión. (Ver Tabla 1).

informes de gestión, se recomiendan no solo para cumplir con los principios de transparencia y rendición de cuentas que le corresponden a la Institución dado su carácter público, sino también como una estrategia para evidenciar -ante los diferentes grupos de la comunidad universitaria y nacional- los alcances y los beneficios del VER-UCR. Se contempla la elaboración de informes anuales en dos niveles:

- Por área académica de gestión: Investigación, Acción Social, Vida Estudiantil, Docencia, Desarrollo Regional y Dirección Superior.

- Institucional: que integrará los resultados y el impacto de la gestión del VER desarrollada en todas las unidades ejecutoras de la UCR.

\section{Reflexión Final}

En el contexto de la UCR es claro el crecimiento y reconocimiento del VER 
Tabla 1

Ejemplos de Indicadores de Gestión VER-UCR

\begin{tabular}{|c|c|c|c|c|c|}
\hline 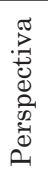 & 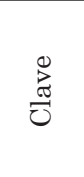 & $\begin{array}{l}\text { ๖ृँ } \\
\text { है } \\
\text { Z }\end{array}$ & 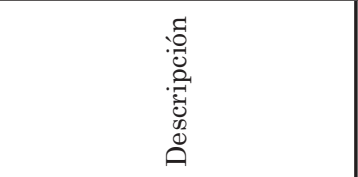 & 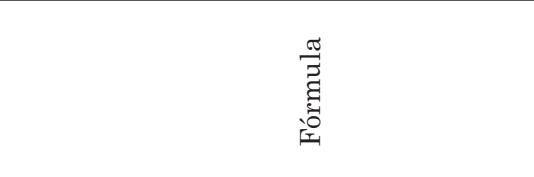 & \\
\hline \multirow{4}{*}{ 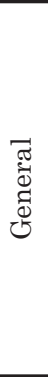 } & \multirow{2}{*}{ G-2 } & \multirow{2}{*}{$\begin{array}{l}\text { Actividades del } \\
\text { VER por sectores }\end{array}$} & \multirow{2}{*}{$\begin{array}{l}\text { Porcentaje de actividades } \\
\text { de VER en el año, por } \\
\text { sector, respecto al total } \\
\text { de actividades de VER } \\
\text { del año }\end{array}$} & Total actividades VER por sector & \multirow{2}{*}{ * 100} \\
\hline & & & & Total actividades VER & \\
\hline & \multirow{2}{*}{ G-5 } & \multirow{2}{*}{$\begin{array}{l}\text { Participación de } \\
\text { unidades ejecu- } \\
\text { toras }\end{array}$} & \multirow{2}{*}{$\begin{array}{l}\text { Porcentaje de unidades } \\
\text { ejecutoras participantes } \\
\text { del VER en el año respec- } \\
\text { to al total de unidades } \\
\text { ejecutoras UCR }\end{array}$} & $\begin{array}{l}\text { Total unidades ejecutoras } \\
\text { participantes en el VER }\end{array}$ & \multirow{2}{*}{ * 100} \\
\hline & & & & Total unidades ejecutoras de la UCR & \\
\hline \multirow{4}{*}{ 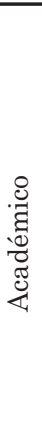 } & \multirow{2}{*}{ A-4 } & \multirow{2}{*}{$\begin{array}{l}\text { Porcentaje de } \\
\text { cambio en activi- } \\
\text { dades académicas } \\
\text { de Educación } \\
\text { Continua (por } \\
\text { unidad ejecutora / } \\
\text { por área) }\end{array}$} & \multirow{2}{*}{$\begin{array}{l}\text { Variación porcentual en } \\
\text { actividades académicas } \\
\text { de Educación Continua } \\
\text { realizadas en el año con } \\
\text { respecto a las realizadas } \\
\text { en el año anterior }\end{array}$} & $\begin{array}{c}\text { Actividades académicas de Educ. } \\
\text { Continua realizadas en el año }\end{array}$ & \multirow{2}{*}{ * 100} \\
\hline & & & & $\begin{array}{l}\text { Actividades académicas de Educ. } \\
\text { Cont. realizadas en el año anterior }\end{array}$ & \\
\hline & \multirow[b]{2}{*}{ A- 8} & \multirow{2}{*}{$\begin{array}{l}\text { Cumplimiento } \\
\text { proyectos de } \\
\text { investigación (por } \\
\text { área) }\end{array}$} & \multirow{2}{*}{$\begin{array}{l}\text { Porcentaje proyectos de } \\
\text { investigación terminados } \\
\text { en el año respecto a los } \\
\text { programados a terminar }\end{array}$} & $\begin{array}{l}\text { Proyectos de investigación } \\
\text { Terminados en el año }\end{array}$ & \multirow{2}{*}{ * 100} \\
\hline & & & & $\begin{array}{l}\text { Proyectos programados a } \\
\text { terminar en el año }\end{array}$ & \\
\hline \multirow{3}{*}{ 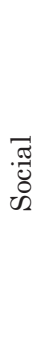 } & $\mathrm{S}-2$ & $\begin{array}{l}\text { Población benefi- } \\
\text { ciada directamente } \\
\text { en Educación } \\
\text { Continua }\end{array}$ & $\begin{array}{l}\text { Número de personas } \\
\text { beneficiadas directa- } \\
\text { mente con actividades de } \\
\text { Educación Continua }\end{array}$ & $\begin{array}{c}\text { Total personas beneficiadas directame } \\
\text { con Educación Continua }\end{array}$ & \\
\hline & \multirow{2}{*}{ S-9 } & \multirow{2}{*}{$\begin{array}{l}\text { Participación del } \\
\text { Ver en actividades } \\
\text { de VENR }\end{array}$} & \multirow{2}{*}{$\begin{array}{l}\text { Porcentaje de actividades } \\
\text { de VENR financiadas con } \\
\text { el VER respecto al total } \\
\text { de actividades de VENR } \\
\text { de la UCR }\end{array}$} & $\begin{array}{c}\text { Número de actividades de VENR } \\
\text { Financiadas con VER }\end{array}$ & \multirow{2}{*}{ * 100} \\
\hline & & & & Total de actividades de VENR-UCR & \\
\hline \multirow{4}{*}{. } & \multirow[b]{2}{*}{ F-2 } & \multirow{2}{*}{$\begin{array}{l}\text { Porcentaje de } \\
\text { cambio en los } \\
\text { ingresos VER }\end{array}$} & \multirow{2}{*}{$\begin{array}{l}\text { Variación porcentual en } \\
\text { los ingresos generados por } \\
\text { el VER en el año respecto } \\
\text { a los generados en el año } \\
\text { anterior. }\end{array}$} & Ingresos generados en el Año & \multirow{2}{*}{ * 100} \\
\hline & & & & $\begin{array}{c}\text { Ingresos generados en el } \\
\text { año anterior }\end{array}$ & \\
\hline & \multirow[t]{2}{*}{ F-10 } & \multirow{2}{*}{$\begin{array}{l}\text { Financiamiento } \\
\text { del } \\
\text { VER al VENR }\end{array}$} & \multirow{2}{*}{$\begin{array}{l}\text { Porcentaje de ingresos del } \\
\text { VER destinados a VENR } \\
\text { respecto a los ingresos } \\
\text { totales VER }\end{array}$} & $\begin{array}{l}\text { Ingresos del VER destinados } \\
\text { a actividades de VENR }\end{array}$ & \multirow{2}{*}{$* 100$} \\
\hline & & & & Ingresos totales VER & \\
\hline
\end{tabular}

Fuente: Arroyo Vengas, Isabel Cristina y Penabad Camacho, Liana. (2008). Impacto del vínculo externo remunerado de la Universidad de Costa Rica sobre su gestión estratégica. San José, Costa Rica: Universidad de Costa Rica. 
como una obligación y una prioridad que se debe atender sobre la base de la solidaridad y la transparencia, criterio compartido plenamente por las otras tres universidades estatales.

A partir de la información recopilada y el respectivo análisis de las cifras que se reportaron para el año 2006, se confirma el importante rol que juegan las actividades de vinculación remunerada desarrolladas por la UCR, principalmente por su impacto social y financiero.

No obstante, se evidenció la necesidad de plantear y ejecutar algunos cambios y ajustes en el nivel de la Conceptualización, Marco Estratégico, Marco Operativo y Seguimiento y Evaluación de Resultados con el propósito de potenciar y optimizar todas las actividades de Vinculación Remunerada; razón por la cual, se ofrece una propuesta que en términos generales podría fortalecer y mejorar la gestión en este importante ámbito, desde los cuatro niveles señalados.

\section{Referencias bibliográficas}

Consejo Nacional de Rectores [CONARE], Oficina de Planificación de la Educación Superior [OPES]. (2005). Plan Nacional de la Educación Superior Universitaria Estatal, [PLANES] 2006-2010. San José, C. R.: CONARE-OPES.

Universidad de Costa Rica, Oficina Jurídica. (2000). Estatuto Orgánico de la Universidad de Costa Rica. Título I: Declaración de principios, propósitos y funciones. En Compendio de Normativa Universitaria ( $2^{\mathrm{a}}$ ed., pp. 22-86). San José, C. R.: Oficina Jurídica, Universidad de Costa Rica.

Universidad de Costa Rica, Vicerrectoría de Administración. (2006). Estados financieros y liquidación presupuestaria al 31 de diciembre de 2006. San José, Costa Rica: Oficina de Administración Financiera. 
